\title{
Time-consistent Optimal Portfolio Strategy for Asset-liability Management under Mean-variance Criterion
}

\author{
Chanjuan $\mathrm{Li}^{1}$, Zhongfei $\mathrm{Li}^{2}, \mathrm{Ke} \mathrm{Fu}^{3}$ \& Haiqing Song ${ }^{3}$ \\ ${ }^{1}$ School of Economics and Management, Inner Mongolia University, Huhhot, China \\ ${ }^{2}$ Sun Yat-sen Business School, Sun Yat-sen University, Guangzhou, China \\ ${ }^{3}$ Lingnan (University) College, Sun Yat-sen University, Guangzhou, China \\ Correspondence: Zhongfei Li, Sun Yat-sen Business School, Sun Yat-sen University, No. 135, Xingang Xi Road, \\ Guangzhou, 510275, China. E-mail: lnslzf@mail.sysu.edu.cn \\ Received: May 3, 2013 \\ doi:10.5430/afr.v2n2p89 \\ Accepted: May 18, 2013 \\ Online Published: May 19, 2013 \\ URL: http://dx.doi.org/10.5430/afr.v2n2p89
}

\begin{abstract}
This paper studies the time-consistent optimal portfolio strategy of an investor with an exogenous liability. Assume that the investor adopts the mean-variance criterion and trades continuously in a market consisting of one risk-free asset and one risky asset; and the price of the risky asset and the value of the exogenous liability are governed by geometric Brownian motions. An extended Hamilton-Jacobi-Bellman equation is derived, and the analytical expressions of the time-consistent optimal portfolio strategy and the mean-variance efficient frontier are obtained. A numerical example is provided to show the results. Our main findings are: (1) introducing an exogenous liability makes the time-consistent optimal portfolio strategy be a stochastic process; (2) the efficient frontier under the time-consistent optimal strategy for asset-liability management is below both the one under the time-consistent optimal strategy in the case of no liability and the one under the pre-commitment optimal strategy for asset-liability management.
\end{abstract}

Keywords: Time-consistent, Asset-liability management, Mean-variance criterion, Portfolio selection, Exogenous liability

\section{Introduction}

For an investor, it is important to seek the best allocation of her/his wealth among a variety of securities so as to achieve her/his objective given in advance. Over the past few decades, many scholars have focused on this problem. Among their works, the mean-variance (MV) portfolio selection pioneered by Markowitz (1952) is the most influential one and provides a fundamental basis for portfolio construction in a single period. After Markowitz's work, it has been difficult for scholars to derive significant results for multi-period MV portfolio selection for a long time. This situation is changed by introducing the embedding technique, initiated by $\mathrm{Li}$ and $\mathrm{Ng}$ (2000) and Zhou and $\mathrm{Li}$ (2000), which is used to transform a nonseparable dynamic multi-period MV problem to the one which can be solved directly by dynamic programming approach. Consequently, the analytical optimal solutions in discrete-time multi-period case and continuous-time case are derived, respectively. Since then, numbers of scholars study this problem in a more realistic market or with more realistic constraints. For instance, Zhou and Yin (2003) and Yin and Zhou (2004) investigate a continuous-time MV portfolio selection problem with regime switching, while Çakmak and Özekici (2006) and Çelikyurt and Özekici (2007) consider a discrete-time multi-period version; Bielecki et al. (2005) study a continuous-time MV portfolio selection problem with bankruptcy prohibition in a market which all the market coefficients are random, while Zhu et al. (2004) investigate the discrete-time multi-period case.

The literature mentioned above does not take account of liability. However, there are some kinds of institutions, for instance, banks and pension funds, which have to consider both sides of the balance sheet. Thus, liability is an essential factor for such institutions. Noticed this point, many scholars have investigated portfolio selection problems with liability. For instance, Sharpe and Tint (1990), for the first time, investigate the single-period asset-liability (AL) management under the MV criterion. Leippold et al. (2004) present a geometric approach to a discrete-time multi-period MV portfolio selection problem with liabilities and derive the analytical solution in a special case. Yi et al. (2008) solve the discrete-time multi-period portfolio problem for AL management with uncertain investment horizon. Chiu and $\mathrm{Li}$ (Chiu and $\mathrm{Li}, 2006$ ) use geometric Brownian motion to govern the exogenous liability and 
solve the continuous-time AL management problem, they derive both the analytical solution and the MV efficient frontier by using stochastic linear-quadratic technique. Employing the technique of Zhou and Yin (2003), Chen et al. (2008) and Xie (2009) solve a continuous-time MV portfolio selection problem with liability and regime switching. The liabilities in Chen, Yang and Yin (2008) and Xie (2009) are governed by geometric Brownian motion and Brownian motion with drift respectively. Recently, Zeng and Li (2011a) and Zeng et al. (2013) use the Lévy process to govern the exogenous liability and investigate continuous-time AL management problems under benchmark and MV criterion in a jump diffusion market by adopting stochastic dynamic programming and duality theory. Noticed that the MV objective function lacks the iterated-expectation property, traditional dynamic programming approach is unable to apply directly to the dynamic portfolio selection problem with MV criterion. The literature mentioned above handles this problem by employing either the martingale method or the embedding technique under the implicit assumption that the decision-makers choose the optimal strategies at an initial time and commit that they will follow the strategies chosen at the initial time in the future. However, because of the lack of the iterated-expectation, MV criterion is actually time-inconsistent. It means that, unless there exists some pre-commitment mechanism, the optimal strategy $u_{0}^{*}$, which represents the optimal solution of MV portfolio selection problem of the investor starting from time $t_{0}$ within the investment horizon $\left[t_{0}, T\right]$, is different from the optimal strategy $u_{1}^{*}$, which is derived by solving the MV portfolio selection problem of the investor with the initial time $t_{1}$ and the investment horizon $\left[t_{1}, T\right]$, where $t_{0}<t_{1}$ and $\mathrm{T}$ is the terminal time of investment horizon. Here, the difference between $u_{0}^{*}$ and $u_{1}^{*}$ means that there exists a subset $\mathcal{T} \subset\left[t_{1}, T\right]$, such that $\forall t \in \mathcal{T}, u_{0}^{*}(t) \neq u_{1}^{*}(t)$. Thus, the optimal strategy of dynamic portfolio selection problem with MV criterion derived by employing the martingale method or the embedding technique under the pre-commitment assumption is referred as pre-commitment strategy. However, it is necessary for rational decision-makers in many situations that the optimal strategy is time-consistent. This is because, at any point of the investment horizon, the investor will choose the strategy which is optimal sitting at current time instead of the one being optimal at an initial time only. Thus, if $\theta_{0}^{*}$ and $\theta_{1}^{*}$ are the time-consistent optimal portfolio strategies for the time $t_{0}$ 's investor and the time $t_{1}$ 's investor under the MV criterion within the investment horizon $\left[t_{0}, T\right]$ and $\left[t_{1}, T\right]$, respectively, where $t_{0}, t_{1}$ and $\mathrm{T}$ are mentioned above, they must satisfy the condition $\theta_{0}^{*}(t)=\theta_{1}^{*}(t), \forall t \in\left[t_{1}, T\right]$. Strotz (1956), for the first time in 1956, formalizes time-consistency and proposes that time-inconsistent problems can be solved either by the pre-commitment strategies or by the time-consistent strategies. It is not until recently that time-consistent stochastic problems attract much attention once again. Ekeland and Pirvu (2008) investigate the Merton portfolio selection problem in the context of non-exponential discounting which gives rise to the time-inconsistency and provide an explicit solution for the case of logarithmic utility. Marín-Solano and Patxot (2012) analyze a model in which the instantaneous utility function and the final function are discounted at different instantaneous discount rates of time preference, and derive a dynamic programming equation whose solutions are time-consistent. Björk and Murgoci (2010) and Björk et al. (2012) view the time-consistent stochastic control problems within a game theoretic framework, and derive an extended Hamilton-Jacobi-Bellman equation to determine the equilibrium strategy and the equilibrium value function. Basak and Chabakauri (2010) solve the dynamic MV portfolio problem within a general incomplete-market economy and derive its time-consistent optimal strategy by employing dynamic programming. Czichowsky (2013) develops a time-consistent formulation of the MV portfolio selection in a general semimartingale setting, and studies both the discrete-time case and the continuous-time case. Wang and Forsyth (2011) develop a numerical scheme for determining a time-consistent optimal strategy for the continuous-time MV portfolio selection problem with any type of constraints to be applied to the investment behavior. Zeng and Li (2011b) derive the analytical time-consistent optimal strategies and the optimal value functions for an investment-reinsurance problem and an investment-only problem for an insurer who holds the MV criterion. Recently, Li et al. (2012) consider the above problems under Heston's stochastic volatility model and also derive the analytical optimal strategies and optimal value functions.

To our knowledge, there is no literature on the time-consistent portfolio strategy of the dynamic AL management problem. However, for an investor whose liability is essential to her/his balance sheet, how to choose the time-consistent portfolio strategy under the MV criterion is an important problem. Therefore, we investigate this meaningful problem in this paper. Specially, we consider an investor who trades continuously in a market consisting of one risk-free asset and one risky asset, and has to take her/his liability into account. Adopting the MV criterion, the investor intends to find out the time-consistent optimal portfolio strategy which will be followed during the whole investment horizon. The rest of the paper is organized as follows. Section 2 describes the assumptions and the 
model. Section 3 provides the extended HJB equation of the problem and the analytical time-consistent optimal portfolio strategy. The MV efficient frontier is computed in Section 4. Section 5 presents a numerical example to illustrate our results. Section 6 concludes this paper. The proofs are provided in the Appendix, which is in section 7.

\section{Assumptions and Modeling}

At the beginning, we consider a filtered complete probability space $\left(\Omega, \mathrm{F},\left\{\mathrm{F}_{t}\right\}_{0 \leq t \leq T}, \mathrm{P}\right)$, where $T$ is a finite constant representing the investment horizon; $\mathrm{F}_{t}$ stands for all the information available at time $t$, and is the augmented filtration generated by 2-dimensional standard Brownian motion $\left\{\left(W^{0}(t), W(t)\right)\right\}$. Assume that all processes introduced below are well defined on this space.

Consider an investor who trades in a financial market consisting of a risk-free asset (bond or bank account) and a risky asset (stock or mutual fund). The price process of the risk-free asset, denoted by $A_{0}(t)$, evolves according to the ordinary differential equation (ODE)

$$
\mathrm{d} A_{0}(t)=r_{0} A_{0}(t) \mathrm{d} t, A_{0}(0)=a_{0},
$$

where $a_{0}$ is the initial price of the risk-free asset; $r_{0}$ is the positive constant interest rate. The price process of the risky asset, denoted by $A(t)$, follows the stochastic differential equation (SDE)

$$
\mathrm{d} A(t)=A(t)[r \mathrm{~d} t+\sigma \mathrm{d} W(t)], A(0)=a,
$$

where $a$ is the initial price of the risky asset; the appreciation rate $r$ and the volatility $\sigma$ are positive constants. Assume that the initial wealth of the investor is $\mathrm{x}_{0}$ and she/he will choose a portfolio strategy $\theta=\{\theta(t)\}_{0 \leq t \leq T}$ to achieve her/his objective, where $\theta(t)$ is the dollar amount of wealth invested in the risky asset at time $t$. When the strategy $\theta$ is adopted, the corresponding wealth process, denoted by $X^{\theta}(t)$, is described as

$$
\mathrm{d} X^{\theta}(t)=\left[r_{0} X^{\theta}(t)+\left(r-r_{0}\right) \theta(t)\right] \mathrm{d} t+\sigma \theta(t) \mathrm{d} W(t), X(0)=x_{0} .
$$

Besides the assets, the investor takes an exogenous liability into account. The "exogenous" means that the liability is not affected by the portfolio, thus it is uncontrollable. The value process of liability $L(t)$ evolves as

$$
\mathrm{d} L(t)=L(t)[\alpha \mathrm{d} t+\beta \mathrm{d} \tilde{W}(t)], L(0)=l_{0},
$$

where $l_{0}$ is the initial value of the liability; $\{\tilde{W}(t)\}$ is a one-dimensional standard Brownian motion defined on the underlying probability space $\left(\Omega, \mathrm{F},\left\{\mathrm{F}_{t}\right\}_{0 \leq t \leq T}, \mathrm{P}\right)$. Denote $\rho$ as the correlation coefficient between $W(t)$ and $\tilde{W}(t)$. According to Koo (1998), $\tilde{W}(t)$ can be expressed as the linear combination of $W(t)$ and $W^{0}(t)$ :

$$
\tilde{W}(t)=\rho W(t)+\sqrt{1-\rho^{2}} W^{0}(t) .
$$

In addition, we define the surplus at time $t$ to be the difference between the asset value and the liability value, as $S^{\theta}(t)=X^{\theta}(t)-L(t)$. Thus the process of $S^{\theta}(t)$ evolves as

$$
\mathrm{d} S^{\theta}(t)=\left[r_{0} S^{\theta}(t)+\left(r_{0}-\alpha\right) L(t)+\left(r-r_{0}\right) \theta(t)\right] \mathrm{d} t+\sigma \theta(t) \mathrm{d} W(t)-\beta L(t) \mathrm{d} \tilde{W}(t), S(0)=s_{0},
$$

where $s_{0}=x_{0}-l_{0} ; L(t)$ is governed by (4).

A strategy $\theta$ is said to be admissible if it satisfies the following conditions: (1) $\forall t \in[0, T], \theta(t)$ is $\mathrm{F}_{t}$ -progressively measurable; (2) $\mathrm{E}\left[\int_{0}^{T}|\theta(t)|^{2} \mathrm{~d} t\right]<\infty$. Denote $\Theta$ as the set of all admissible strategies.

Assume that the investor adopts the MV criterion to guide her/his investment. That is, the optimization problem of the investor can be stated as

$$
\max _{\theta \in \Theta} \quad \mathrm{E}_{0, s_{0}, l_{0}}\left[S^{\theta}(T)\right]-\frac{\lambda}{2} \operatorname{Var}_{0, s_{0}, l_{0}}\left[S^{\theta}(T)\right]
$$

$$
\text { subject to (4) and (5), }
$$

where $\mathrm{E}_{t, s, l}[\cdot]=\mathrm{E}\left[\cdot \mid S^{\theta}(t)=s, L(t)=l\right] ; \lambda \in(0,+\infty)$ is a constant and represents the risk aversion coefficient of the investor.

The aim of our work is to derive the time-consistent optimal strategy rather than the pre-commitment optimal one for 
this problem. Actually, the MV optimization problem with pre-commitment means that the objective function of the investor is always

$$
U_{0}\left(0, s_{0}, l_{0}\right)=\mathrm{E}_{0, s_{0}, l_{0}}\left[S^{\theta}(T)\right]-\frac{\lambda}{2} \operatorname{Var}_{0, s_{0}, l_{0}}\left[S^{\theta}(T)\right]
$$

In contrast, the investor in the MV optimization problem without pre-commitment changes her/his objective function according to the current status, i.e. the objective function at time $t$ is

$$
U(t, s, l)=\mathrm{E}_{t, s, l}\left[S^{\theta}(T)\right]-\frac{\lambda}{2} \operatorname{Var}_{t, s, l}\left[S^{\theta}(T)\right] .
$$

It can be understood that the investor with pre-commitment mechanism pre-commits herself/himself to the target determined at the initial time and does not update it, while the one without pre-commitment updates her/his objective at any subsequent time. Consequently, the MV optimization problem with pre-commitment can be regarded as a static optimization problem in the sense of static objective function, while the one without pre-commitment can be regarded as a dynamic MV optimization problem because of its dynamic updating of the objective.

\section{Solution}

By means of a recursive formulation which is based on stochastic dynamic programming, we can solve the MV optimization problem without pre-commitment. Our method is analogous to that of Basak and Chabakauri (2010).

Based on the law of total variance (see Weiss (2005)), we obtain the following recursive formula of the time $t$ 's objective function:

$$
\begin{aligned}
& U(t, s, l)=\mathrm{E}_{t, s, l}\left[\mathrm{E}_{t+\tau, S^{\theta}(t+\tau), L(t+\tau)}\left[S^{\theta}(T)\right]-\frac{\lambda}{2} \operatorname{Var}_{t+\tau, S^{\theta}(t+\tau), L(t+\tau)}\left[S^{\theta}(T)\right]\right] \\
& -\frac{\lambda}{2} \operatorname{Var}_{t, s, l}\left[\mathrm{E}_{t+\tau, S^{\theta}(t+\tau), L(t+\tau)}\left[S^{\theta}(T)\right]\right] \\
& =\mathrm{E}_{t, s, l}\left[\mathrm{U}\left(t+\tau, S^{\theta}(t+\tau), L(t+\tau)\right)\right]-\frac{\lambda}{2} \operatorname{Var}_{t, s, l}\left[\mathrm{E}_{t+\tau, S^{\theta}(t+\tau), L(t+\tau)}\left[S^{\theta}(T)\right]\right], \forall \tau>0 .
\end{aligned}
$$

The adjustment term $-\frac{\lambda}{2} \operatorname{Var}_{t, s, l}\left[\mathrm{E}_{t+\tau, S^{\theta}(t+\tau), L(t+\tau)}\left[S^{\theta}(T)\right]\right]$ reflects the incentive of the time $t+\tau$ 's investor to change the time $t$ 's optimal strategy. Because of this term, we determine the optimal portfolio strategy by backward induction as in Basak and Chabakauri (2010). The backward induction is such a method which assumes that at each moment the investor chooses the strategy which is actually optimal at any subsequent time and thus will be followed in the future. Thus, the investor will follow the optimal portfolio strategy derived by backward induction during the whole investment horizon, which means that it is a time-consistent optimal strategy.

Given the time-consistent optimal strategy $\theta^{*}(s), s \in[t, T]$, the optimal value function $J$ is defined as

$$
J\left(t, S^{\theta}(t), L(t)\right)=\mathrm{E}_{t, S^{\theta}(t), L(t)}\left[S^{\theta^{*}}(T)\right]-\frac{\lambda}{2} \operatorname{Var}_{t, S^{\theta}(t), L(t)}\left[S^{\theta^{*}}(T)\right],
$$

where the terminal surplus $S^{\theta^{*}}(T)$ is computed under the optimal strategy $\theta^{*}(s), s \in[t, T]$. The recursive relationship for $J\left(t, S^{\theta}(t), L(t)\right)$ is necessary and can be derived as follows. Suppose $\tau>0$ is a decision-making interval which means that the investor will follow the optimal strategy $\theta^{*}(s)$ within interval $[t+\tau, T]$, while she/he will search for the optimal strategy within interval $[t, t+\tau]$. According to the recursive formula (8) of objective function $U(t, s, l)$,

$$
\begin{aligned}
& J\left(t, S^{\theta}(t), L(t)\right) \\
& =\max _{\theta(s): s \in[t, T]}\left\{\mathrm{E}_{t, s, l}\left[S^{\theta}(T)\right]-\frac{\lambda}{2} \operatorname{Var}_{t, s, l}\left[S^{\theta}(T)\right]\right\} \\
& =\max _{\theta(s): s \in[t, t+\tau]}\left\{\max _{\theta(s): s \in[t+\tau, T]}\left\{\mathrm{E}_{t, s, l}\left[\mathrm{U}\left(t+\tau, S^{\theta}(t+\tau), L(t+\tau)\right)\right]-\frac{\lambda}{2} \operatorname{Var}_{t, s, l}\left[\mathrm{E}_{t+\tau, S^{\theta}(t+\tau), L(t+\tau)}\left[S^{\theta}(T)\right]\right]\right\}\right\} \\
& =\max _{\theta(s): s \in[t, t+\tau]}\left\{\mathrm{E}_{t, s, l}\left[J\left(t+\tau, S^{\theta}(t+\tau), L(t+\tau)\right)\right]-\frac{\lambda}{2} \operatorname{Var}_{t, s, l}\left[\mathrm{E}_{t+\tau, S^{\theta}(t+\tau), L(t+\tau)}\left[S^{\theta^{*}}(T)\right]\right]\right\}
\end{aligned}
$$


where $J\left(t+\tau, S^{\theta}(t+\tau), L(t+\tau)\right)$ and $\mathrm{E}_{t+\tau, S^{\theta}(t+\tau), L(t+\tau)}\left[S^{\theta^{*}}(T)\right]$ on $[t+\tau, T]$ are computed under the strategy $\theta^{*}(s), s \in[t+\tau, T]$, and the states $S^{\theta}(t+\tau)$ as well as $L(t+\tau)$. Thus, the problem of the investor at time $t$ is to find a portfolio strategy $\theta(s)$, for $s \in[t, t+\tau]$ such that $\mathrm{E}_{t, s, l}\left[J\left(t+\tau, S^{\theta}(t+\tau), L(t+\tau)\right)\right]-\frac{\lambda}{2} \operatorname{Var}_{t, s, l}\left[\mathrm{E}_{t+\tau, S^{\theta}(t+\tau), L(t+\tau)}\left[S^{\theta^{*}}(T)\right]\right]$ is maximized. (10) means that, on the one hand, within interval $[t+\tau, T]$, the time $t$ 's investor will follow the strategy $\theta^{*}(s)$, which is the time-consistent optimal strategy for the time $t+\tau$ 's investor within the investment horizon $[t+\tau, T]$; on the other hand, because of the term $\operatorname{Var}_{t, s, l}\left[\mathrm{E}_{t+\tau, S^{\theta}(t+\tau), L(t+\tau)}\left[S^{\theta^{*}}(T)\right]\right]$, strategy $\theta^{*}(s), s \in[t+\tau, T]$, may not be the optimal strategy for the time $t$ 's investor. Thus, given by the time-consistent optimal strategy $\theta^{*}(s), s \in[t+\tau, T]$, the time $t$ 's investor should find a optimal strategy in the sense of (10) within interval $[t, t+\tau]$.

Lemma 3.1

Assume that $\theta^{*}(s), s \in[t, T]$, is the time-consistent optimal portfolio strategy of time $t$ 's investor in problem (6). Define

$$
f\left(t, S^{\theta}(t), L(t)\right)=\mathrm{E}_{t, S^{\theta}(t), L(t)}\left[S^{\theta^{*}}(T)\right]-S^{\theta}(T) \exp \left(r_{0}(T-t)\right)
$$

where $S^{\theta^{*}}(T)$ is the terminal surplus under $\theta^{*}(s), s \in[t, T]$. Then $f\left(t, S^{\theta}(t), L(t)\right)$ can be computed as

$$
f\left(t, S^{\theta}(t), L(t)\right)=\mathrm{E}_{t, S^{\theta}(t), L(t)}\left[\int_{t}^{T} \exp \left(r_{0}(T-s)\right)\left[\left(r_{0}-\alpha\right) L(s)+\left(r-r_{0}\right) \theta^{*}(s)\right] \mathrm{ds}\right] .
$$

Theorem 3.1

The optimal value function $J\left(t, S^{\theta}(t), L(t)\right)$ of problem (6) satisfies the following recursive formula:

$$
0=\max _{\theta(t)} \mathrm{E}_{t, S^{\theta}(t) L(t)}\left[\mathrm{d} J\left(t, S^{\theta}(t), L(t)\right)\right]-\frac{\lambda}{2} \operatorname{Var}_{t, S^{\theta}(t) L(t)}\left[\mathrm{d} f\left(t, S^{\theta}(t), L(t)\right)+\mathrm{d}\left(S^{\theta}(T) \exp \left(r_{0}(T-t)\right)\right)\right]
$$

with terminal condition $J(T, S(T), L(T))=S(T)$. Furthermore, $J\left(t, S^{\theta}(t), L(t)\right)$ can be represented as

$$
J\left(t, S^{\theta}(t), L(t)\right)=S^{\theta}(t) \exp \left(r_{0}(T-t)\right)+\tilde{J}(t, L(t)) .
$$

Meanwhile, the time-consistent optimal strategy $\theta^{*}(t)$ and $f\left(t, S^{\theta}(t), L(t)\right)$ do not depend on time t's surplus $S^{\theta}(t)$ and are functions of $L(t)$ and $t$ only.

Remark 3.1

Theorem 3.1 provides the HJB equation (13) in the differential form and the separability property of $J, f$ and the optimal strategy $\theta^{*} . f\left(t, S^{\theta}(t), L(t)\right)$, which can be written as $f(t, L(t))$, represents the expected total gains or losses from the optimal strategy over the horizon $[t, T]$. Furthermore, since $f\left(t, S^{\theta}(t), L(t)\right)$ is evaluated under the optimal strategy, the term $\mathrm{d} f\left(t, S^{\theta}(t), L(t)\right)$ in (13) is not affected by the control $\theta(t)$.

Theorem 3.2

The time-consistent optimal portfolio strategy of problem (6) is given by

Remark 3.2

$$
\theta^{*}(t)=\frac{r-r_{0}}{\lambda \sigma^{2}} \exp \left(-r_{0}(T-t)\right)+\frac{\beta \rho}{\sigma} L(t)-\frac{\partial f(t, L(t))}{\partial L(t)} \frac{\beta \rho}{\sigma} L(t) \exp \left(-r_{0}(T-t)\right), t \in[0, T] .
$$

Substituting $\theta^{*}(t)$ given by (15) into (12) yields the computation formula for $f(t, L(t))$ as

$$
\begin{aligned}
& f(t, L(t)) \\
& =\mathrm{E}_{t, S^{\theta}(t), L(t)}\left[\int_{t}^{T} \exp \left(r_{0}(T-s)\right)\left(r_{0}-\alpha+\frac{r-r_{0}}{\sigma} \beta \rho\right) L(s) \mathrm{ds}\right]+\mathrm{E}_{t, S^{\theta}(t), L(t)}\left[\frac{1}{\lambda}\left(\frac{r-r_{0}}{\sigma}\right)^{2} \mathrm{ds}\right] \\
& -\mathrm{E}_{t, S^{\theta}(t), L(t)}\left[\int_{t}^{T} \frac{\partial f(s, L(s))}{\partial L(s)} \frac{\beta \rho}{\sigma}\left(r-r_{0}\right) L(s) \mathrm{ds}\right] .
\end{aligned}
$$


In order to obtain the explicit form of the solution, we have to find a new probability measure under which $f(t, L(t))$ can be computed directly.

Theorem 3.3

$f(t, L(t))$ can be expressed as

$$
f(t, L(t))=\frac{1}{\lambda}\left(\frac{r-r_{0}}{\sigma}\right)^{2}(T-t)+\mathrm{E}_{t, s, l}^{*}\left[\int_{t}^{T} \exp \left(r_{0}(T-u)\right)\left(r_{0}-\alpha+\frac{r-r_{0}}{\sigma} \beta \rho\right) L(u) \mathrm{du}\right],
$$

where $\mathrm{E}_{t, s, l}^{*}[\cdot]=\mathrm{E}^{*}\left[\cdot \mid S^{\theta}(t)=s, L(t)=l\right]$, denotes the expectation under the unique measure $P^{*}$ given by the Radon-Nikodym derivative

$$
\frac{\mathrm{d} P^{*}}{\mathrm{~d} P}=\exp \left(-\frac{1}{2} \int_{0}^{T}\left(\frac{r-r_{0}}{\sigma}\right)^{2} \mathrm{~d} s-\int_{0}^{T} \frac{r-r_{0}}{\sigma} \mathrm{d} W(s)\right)
$$

The time-consistent optimal portfolio strategy $\theta^{*}$ is given by

$$
\theta^{*}(t)=\frac{r-r_{0}}{\lambda \sigma^{2}} \exp \left(-r_{0}(T-t)\right)+\frac{\beta \rho}{\sigma} L(t) \exp \left(\left(\alpha-\frac{r-r_{0}}{\sigma} \beta \rho-r_{0}\right)(T-t)\right), t \in[0, T] .
$$

The methods of proofs of the above theorems are analogous to Basak and Chabakauri (2010) and the proofs are presented in the appendix.

Remark 3.3

According to (19), we have the following conclusions. First of all, $\theta^{*}$ is a decreasing function of $\lambda$. Thus, the bigger the $\lambda$ is, i.e., the more risk averse the investor is, the less the fund is invested in the risky asset. This agrees with the intuition. Second, in the case of no liability, the time-consistent optimal strategy is reduced to $\theta^{*}(t)=\frac{r-r_{0}}{\lambda \sigma^{2}} \exp \left(-r_{0}(T-t)\right)$, which is coincident with the strategies given by the simplest case of Bjork and Murgoci (2010) and equation (41) of Basak and Chabakauri (2010). We observe that, comparing with the case of no liability, the time-consistent optimal strategy of the case with an exogenous liability is stochastic while the one of the case of no liability is deterministic. Third, following the time-consistent optimal strategy, the investor with an exogenous liability purchases more risky asset than the one without liability does.

Remark 3.4

Comparing our time-consistent optimal portfolio strategy $\theta^{*}$ given by (19) with the optimal portfolio strategy $u^{*}$ derived by Chiu and $\mathrm{Li}$ (2006), we have the conclusion that strategy $u^{*}$ is not time-consistent for the following reason.

As assumed, there are one risk-free asset and one risky asset in the market, while the investor is responsible for an exogenous liability. Furthermore, suppose that the correlation coefficient $\rho=1$. In our settings, the optimal strategy of Chiu and $\mathrm{Li}(2006)$ is

$$
\begin{aligned}
& u^{*}(t, s, l)=\frac{\omega}{2 \lambda} \frac{r-r_{0}}{\sigma^{2}} \exp \left(-r_{0}(T-t)\right) \\
& +\frac{s\left(r-r_{0}\right)}{\sigma^{2}}+l\left(-\frac{r-r_{0}}{\sigma^{2}}+\frac{r-r_{0}+\sigma \beta}{\sigma^{2}} \exp \left(\left(\alpha-r_{0}-\frac{r-r_{0}}{\sigma} \beta\right)(T-t)\right)\right),
\end{aligned}
$$

where $\omega=\left[1+2 \lambda\left(A_{1} s_{0}+\left(A_{1}-A_{2}\right) l_{0}\right)\right] /(1-\Delta)$ is the auxiliary parameter, which is generated by employing the embedding technique of $\mathrm{Li}$ and $\mathrm{Ng}$ (2000), with

$$
\begin{aligned}
& A_{1}=\exp \left(\left(r_{0}-\frac{\left(r-r_{0}\right)^{2}}{\sigma^{2}}\right)\left(T-t_{0}\right)\right), A_{2}=\exp \left(\left(\alpha-\frac{\left(r-r_{0}\right)\left(r-r_{0}+\sigma \beta\right)}{\sigma^{2}}\right)\left(T-t_{0}\right)\right), \\
& \Delta=1-\exp \left(-\frac{\left(r-r_{0}\right)^{2}}{\sigma^{2}}\left(T-t_{0}\right)\right) .
\end{aligned}
$$

Noted that, $t_{0}$ is the initial time of investment horizon, which is equal to 0 in the model of Chiu and $\mathrm{Li}(2006)$. As a result, if the investor enters the market at time $t_{1}, t_{0}<t_{1}<T$, i.e. the initial time is $t_{1}$ rather than $t_{0}$, the coefficients $A_{1}, A_{2}$ and $\Delta$ all change. As a result, the auxiliary parameter $\omega$ change accordingly. The ultimate result is that the optimal portfolio strategy $u^{*}$ changes. Let $u_{0}^{*}$ and $u_{1}^{*}$ denote the optimal strategies of the 
investors at time $t_{0}$ and $t_{1}$, respectively. For the investor at time $t, t_{0}<t_{1}<t<T, u_{0}^{*}(t) \neq u_{1}^{*}(t)$. Consequently, the strategy given by Chiu and Li (2006) is actually a pre-commitment optimal strategy, but not time-consistent.

\section{MV Efficient Frontier}

In this section, we compute the MV efficient frontier. Let $S^{*}(t)$ be the surplus process under the time-consistent optimal portfolio strategy $\theta^{*}(t)$ given by (19). Substituting (19) into (5) yields

$$
\begin{aligned}
& \mathrm{d} S^{*}(t)=\left[r_{0} S^{*}(t)+F_{1}(t) L(t)+\frac{1}{\lambda} F_{2}(t)\right] \mathrm{d} t+\frac{r-r_{0}}{\lambda \sigma^{2}} \exp \left(-r_{0}(T-t)\right) \mathrm{d} W(t) \\
& +L(t) \beta \rho \exp \left(\left(\alpha-\frac{r-r_{0}}{\sigma} \beta \rho-r_{0}\right)(T-t)\right) \mathrm{d} W(t)-L(t) \beta \mathrm{d} \tilde{W}(t), S^{*}(0)=s_{0},
\end{aligned}
$$

where $F_{1}(t)=r_{0}-\alpha+\frac{r-r_{0}}{\sigma} \beta \rho \exp \left(\left(\alpha-\frac{r-r_{0}}{\sigma} \beta \rho-r_{0}\right)(T-t)\right), F_{2}(t)=\frac{\left(r-r_{0}\right)^{2}}{\sigma^{2}} \exp \left(-r_{0}(T-t)\right)$. Applying Itô formula to $L(t)^{2}, S^{*}(t) L(t)$ and $S^{*}(t)^{2}$, we have

$$
\begin{aligned}
& \mathrm{d} L(t)^{2}=\left(2 \alpha+\beta^{2}\right) L(t)^{2} \mathrm{~d} t+2 \beta L(t)^{2} \mathrm{~d} \tilde{W}(t), L(0)^{2}=l_{0}^{2}, \\
& \mathrm{~d}\left(S^{*}(t) L(t)\right)=\left[\left(\alpha+r_{0}\right) S^{*}(t) L(t)+F_{3}(t) L(t)^{2}+\frac{1}{\lambda} F_{4}(t) L(t)\right] \mathrm{d} t+\beta S^{*}(t) L(t) \mathrm{d} \tilde{W}(t) \\
& +\frac{r-r_{0}}{\lambda \sigma} L(t) \exp \left(-r_{0}(T-t)\right) \mathrm{d} W(t)+L(t)^{2} \beta \rho \exp \left(\left(\alpha-\frac{r-r_{0}}{\sigma} \beta \rho-r_{0}\right)(T-t)\right) \mathrm{d} W(t) \\
& -L(t)^{2} \beta \mathrm{d} \tilde{W}(t), S^{*}(0) L(0)=s_{0} l_{0}, \\
& \mathrm{~d} S^{*}(t)^{2}=\left[2 r_{0} S^{*}(t)^{2}+F_{5}(t) S^{*}(t) L(t)+\frac{1}{\lambda} F_{6}(t) S^{*}(t)+F_{7}(t) L(t)^{2}+\frac{1}{\lambda} F_{8}(t) L(t)+\frac{1}{\lambda^{2}} F_{9}(t)\right] \mathrm{d} t \\
& +2 \frac{r-r_{0}}{\lambda \sigma} S^{*}(t) \exp \left(-r_{0}(T-t)\right) \mathrm{d} W(t)+2 \beta \rho S^{*}(t) L(t) \exp \left(\left(\alpha-\frac{r-r_{0}}{\sigma} \beta \rho-r_{0}\right)(T-t)\right) \mathrm{d} W(t) \\
& -2 \beta S^{*}(t) L(t) \mathrm{d} \tilde{W}(t), S^{*}(0)^{2}=s_{0}^{2} .
\end{aligned}
$$

where

$$
\begin{aligned}
& F_{3}(t)=r_{0}-\alpha+\frac{r-r_{0}}{\sigma} \beta \rho \exp \left(\left(\alpha-\frac{r-r_{0}}{\sigma} \beta \rho-r_{0}\right)(T-t)\right)+\beta^{2} \rho^{2} \exp \left(\left(\alpha-\frac{r-r_{0}}{\sigma} \beta \rho-r_{0}\right)(T-t)\right)-\beta^{2}, \\
& F_{4}(t)=\frac{\left(r-r_{0}\right)^{2}}{\sigma^{2}} \exp \left(-r_{0}(T-t)\right)+\frac{r-r_{0}}{\sigma} \beta \rho \exp \left(-r_{0}(T-t)\right), \\
& F_{5}(t)=2\left(r_{0}-\alpha\right)+2 \frac{r-r_{0}}{\sigma} \beta \rho \exp \left(\left(\alpha-\frac{r-r_{0}}{\sigma} \beta \rho-r_{0}\right)(T-t)\right), \\
& F_{6}(t)=\frac{2\left(r-r_{0}\right)^{2}}{\sigma^{2}} \exp \left(-r_{0}(T-t)\right), \\
& F_{7}(t)=\beta^{2} \rho^{2} \exp \left(2\left(\alpha-\frac{r-r_{0}}{\sigma} \beta \rho-r_{0}\right)(T-t)\right)+\beta^{2}-2 \beta^{2} \rho^{2} \exp \left(\left(\alpha-\frac{r-r_{0}}{\sigma} \beta \rho-r_{0}\right)(T-t)\right), \\
& F_{8}(t)=2 \frac{r-r_{0}}{\sigma} \beta \rho\left(\exp \left(\left(\alpha-\frac{r-r_{0}}{\sigma} \beta \rho-2 r_{0}\right)(T-t)\right)-\exp \left(-r_{0}(T-t)\right)\right) \\
& F_{9}(t)=\frac{\left(r-r_{0}\right)^{2}}{\sigma^{2}} \exp \left(-2 r_{0}(T-t)\right),
\end{aligned}
$$

Noted that $\mathrm{E}[L(t)]=l_{0} \exp (\alpha t)$, where $\mathrm{E}[\cdot]$ is abbreviation for $\mathrm{E}_{0, s_{0}, l_{0}}[\cdot]$, taking expectation on the both sides of (22) at time 0 yields

$$
\mathrm{dE}\left[S^{*}(t)\right]=\left(\mathrm{E}\left[S^{*}(t)\right] r_{0}+F_{1}(t) l_{0} \exp (\alpha t)+\frac{1}{\lambda} F_{2}(t)\right) \mathrm{d} t, \mathrm{E}\left[S^{*}(0)\right]=s_{0} .
$$

The solution of ODE (26) is 


$$
\mathrm{E}\left[S^{*}(t)\right]=H_{1}(t)+\frac{1}{\lambda} H_{2}(t)
$$

where

$$
\begin{aligned}
& H_{1}(t)=x_{0} \exp \left(r_{0} t\right)+l_{0} \exp \left(r_{0} t\right) \int_{0}^{t} F_{1}(s) \exp \left(\left(\alpha-r_{0}\right) s\right) \mathrm{d} s, \\
& H_{2}(t)=\exp \left(r_{0} t\right) \int_{0}^{t} \exp \left(-r_{0} s\right) F_{2}(s) \mathrm{d} s .
\end{aligned}
$$

According to (23), $\mathrm{E}\left[L(t)^{2}\right]=l_{0}^{2} \exp \left(\left(2 \alpha+\beta^{2}\right) t\right)$. Taking expectation on the both sides of (24) at time 0 yields

$$
\begin{aligned}
& \mathrm{dE}\left(S^{*}(t) L(t)\right)=\left[\left(\alpha+r_{0}\right) \mathrm{E}\left[S^{*}(t) L(t)\right]+F_{3}(t) l_{0}^{2} \exp \left(\left(2 \alpha+\beta^{2}\right) t\right)+\frac{1}{\lambda} F_{4}(t) l_{0} \exp (\alpha t)\right] \mathrm{d} t, \\
& \mathrm{E}\left[S^{*}(0) L(0)\right]=s_{0} l_{0} .
\end{aligned}
$$

The solution of (29) is

$$
\mathrm{E}\left(S^{*}(t) L(t)\right)=H_{3}(t)+\frac{1}{\lambda} H_{4}(t)
$$

where

$$
\begin{aligned}
& H_{3}(t)=x_{0} l_{0} \exp \left(\left(\alpha+r_{0}\right) t\right)+l_{0}^{2} \exp \left(\left(\alpha+r_{0}\right) t\right) \int_{0}^{t} F_{3}(s) \exp \left(\left(\alpha+\beta^{2}-r_{0}\right) s\right) \mathrm{d} s, \\
& H_{4}(t)=l_{0} \exp \left(\left(\alpha+r_{0}\right) t\right) \int_{0}^{t} \exp \left(-r_{0} s\right) F_{4}(s) \mathrm{d} s .
\end{aligned}
$$

Taking expectation on the both sides of (25) at time 0 yields

$$
\mathrm{dE}\left(S^{*}(t)^{2}\right)=\left[\begin{array}{l}
2 r_{0} \mathrm{E}\left[S^{*}(t)^{2}\right]+F_{5}(t) \mathrm{E}\left[S^{*}(t) L(t)\right]+\frac{1}{\lambda} F_{6}(t) \mathrm{E}\left[S^{*}(t)\right]+F_{7}(t) \mathrm{E}\left[L(t)^{2}\right] \\
+\frac{1}{\lambda} F_{8}(t) \mathrm{E}[L(t)]+\frac{1}{\lambda^{2}} F_{9}(t)
\end{array}\right] \mathrm{d} t,
$$

$\mathrm{E}\left[S^{*}(0)^{2}\right]=s_{0}^{2}$.

Substituting (27), (30), $\mathrm{E}[L(t)]$ and $\mathrm{E}\left[L(t)^{2}\right]$ into the above equation, we have

where

$$
\mathrm{E}\left[S^{*}(t)^{2}\right]=H_{5}(t)+\frac{1}{\lambda} H_{6}(t)+\frac{1}{\lambda^{2}} H_{7}(t),
$$

$$
\begin{gathered}
H_{5}(t)=s_{0}^{2} \exp \left(2 r_{0} t\right)+\exp \left(2 r_{0} t\right) \int_{0}^{t} \exp \left(-2 r_{0} s\right)\left[F_{5}(s) H_{3}(s)+l_{0}^{2} F_{7}(s) \exp \left(\left(2 \alpha+\beta^{2}\right) s\right)\right] \mathrm{d} s, \\
H_{6}(t)=\exp \left(2 r_{0} t\right) \int_{0}^{t} \exp \left(-2 r_{0} s\right)\left[F_{5}(s) H_{4}(s)+F_{6}(s) H_{1}(s)+l_{0} F_{8}(s) \exp (\alpha s)\right] \mathrm{d} s \\
H_{7}(t)=\exp \left(2 r_{0} t\right) \int_{0}^{t} \exp \left(-2 r_{0} s\right)\left[F_{6}(s) H_{2}(s)+F_{9}(s)\right] \mathrm{d} s,
\end{gathered}
$$

Consequently, the variance of the surplus under the time-consistent optimal portfolio strategy is

$$
\operatorname{Var}\left[S^{*}(t)\right]=\left(H_{5}(t)-H_{1}(t)^{2}\right)+\frac{1}{\lambda}\left(H_{6}(t)-2 H_{1}(t) H_{2}(t)\right)+\frac{1}{\lambda^{2}}\left(H_{7}(t)-H_{2}(t)^{2}\right) .
$$

According to (27), $1 / \lambda=\left[\mathrm{E}\left[S^{*}(t)\right]-H_{1}(t)\right] / H_{2}(t)$. Substituting the expression of $\lambda$ into (35), we have the closed-form of the efficient frontier presented in the following theorem.

Theorem 4.1

The efficient frontier of problem (6) under the time-consistent optimal portfolio strategy $\theta^{*}(t)$ is

$$
\begin{aligned}
& \operatorname{Var}\left[S^{*}(T)\right]=\frac{H_{7}(T)-H_{2}(T)^{2}}{H_{2}(T)^{2}}\left(\mathrm{E}\left[S^{*}(T)\right]-H_{1}(T)\right)^{2} \\
& +\frac{H_{6}(T)-2 H_{1}(T) H_{2}(T)}{H_{2}(T)}\left(\mathrm{E}\left[S^{*}(T)\right]-H_{1}(T)\right)+\left(H_{5}(T)-H_{1}(T)^{2}\right),
\end{aligned}
$$

where $H_{1}(T), H_{2}(t), H_{5}(T), H_{6}(T), H_{7}(t)$ are given by (28) and (32) - (34).

Remark 4.1 
After calculation, $H_{2}(T)=\frac{\left(r-r_{0}\right)^{2} T}{\sigma^{2}}, H_{7}(T)=\frac{\left(r-r_{0}\right)^{4} T^{2}}{\sigma^{4}}+\frac{\left(r-r_{0}\right)^{2} T}{\sigma^{2}}$, thus,

$\left(H_{7}(T)-H_{2}(T)^{2}\right) / H_{2}(T)^{2}=1 / H_{2}(T)>0$. Therefore, the efficient frontier given by (36) is a parabola in $\operatorname{Var}\left[S^{*}(T)\right]-\mathrm{E}\left[S^{*}(T)\right]$ plane.

Remark 4.2

Denote $S^{u^{*}}(T)$ as the terminal surplus under strategy $u^{*}$ given by (20). The efficient frontier under strategy $u^{*}$ is $\operatorname{Var}\left[S^{u^{*}}(T)\right]=\frac{1-\Delta}{\Delta}\left[\mathrm{E}\left[S^{u^{*}}(T)\right]-\left(s_{0} \exp \left(r_{0} T\right)+l_{0} \exp \left(r_{0} T\right)-l_{0} \exp \left(\left(\alpha-\frac{r-r_{0}}{\sigma} \beta\right) T\right)\right)\right]^{2}$,

where $\Delta$ is given by $(21)$.

\section{Numerical Example}

This section provides a numerical example to illustrate our results. Of course, using real time-series variables may help demonstrate the proposed solution procedure, but our assessment shows that similar results will be obtained by doing so.

Consider an investor whose initial wealth is $x_{0}=2$ and initial liability is $l_{0}=1$. Throughout this section, unless otherwise noted, the basic parameters are given by $r_{0}=0.06, r=0.2, \sigma=0.08, \beta=0.2, \rho=0.5, T=7$

5.1 Impact of the parameters on the optimal strategy

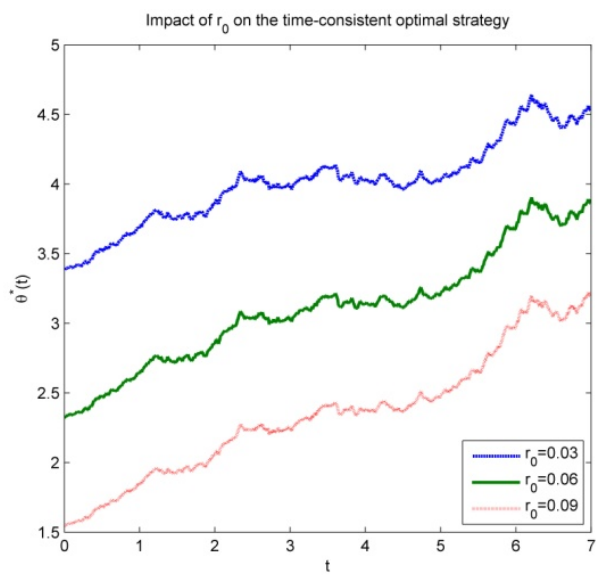

(a)

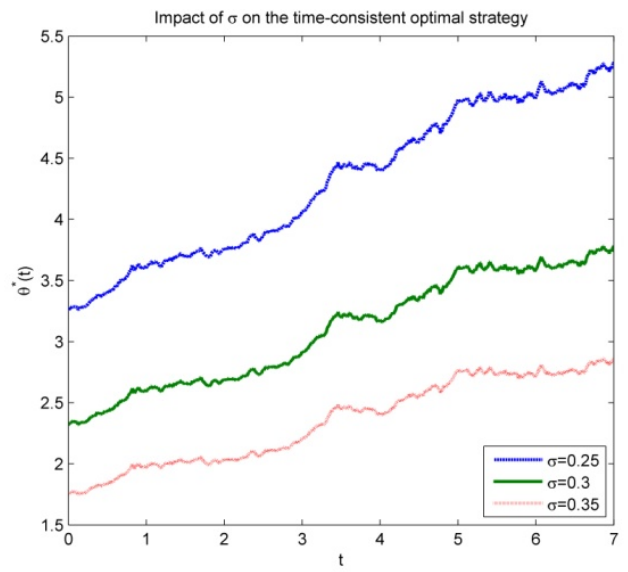

(c)

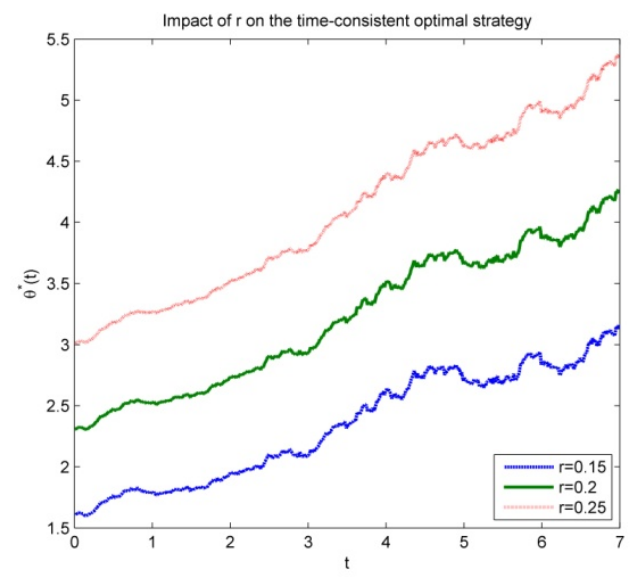

(b)

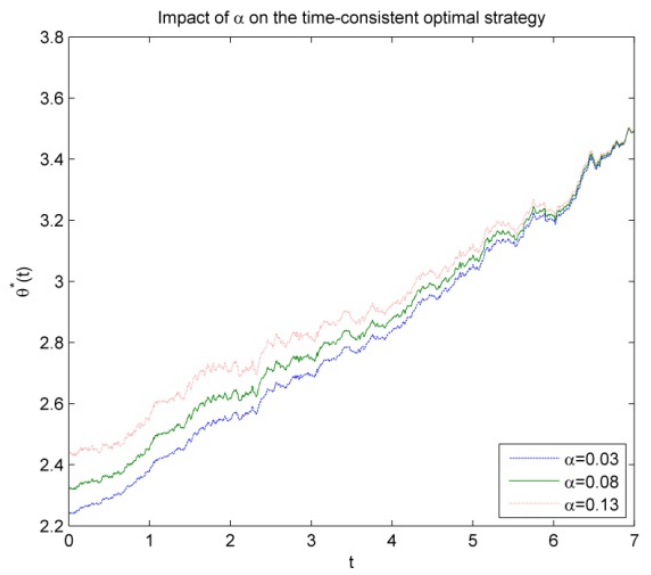

(d) 


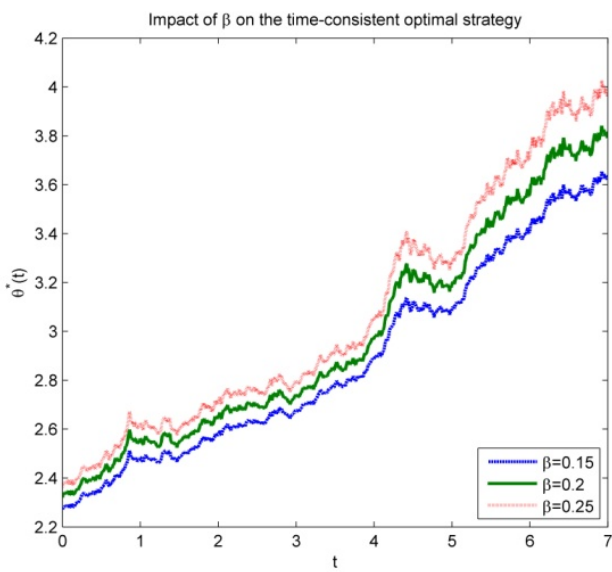

(e)

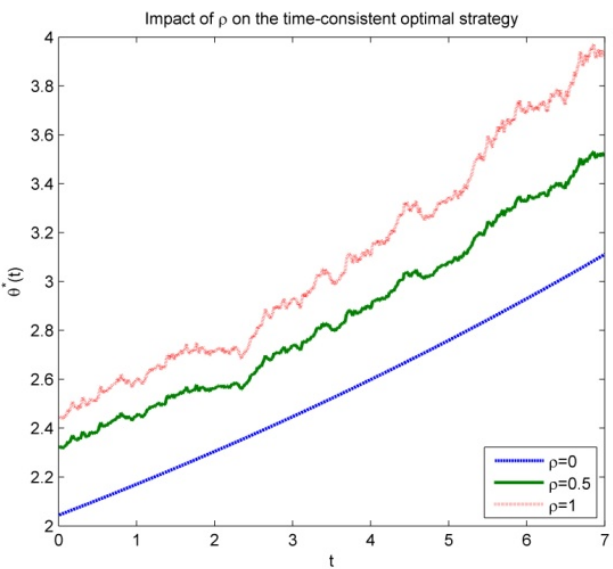

(f)

Figure 1. Impact of the parameters on the time-consistent optimal strategy

After carrying out multiple simulations, we try to get a clearer set of pictures to illustrate the impact of the parameters on the time-consistent optimal strategy. In Figure 1, for the parameters involving the assets, (a) and (c) shows that the larger the interest rate of the risk-free asset $r_{0}$ or the volatility of the risky asset $\sigma$ is, the less money the investor invests in the risky asset; (b) illustrates that the time-consistent optimal strategy $\theta^{*}(t)$ increases as the appreciation rate of the risky asset $r$ increases. For the parameters involving the exogenous liability, (d) shows that $\theta^{*}(t)$ increases as the appreciation rate of the liability $\alpha$ increases; (e) illustrates that the larger the volatility of the liability $\beta$ is, the more money the investor invests in the risky asset. (f) indicates that $\theta^{*}(t)$ increases with the increasing correlation coefficient $\rho$; (f) also demonstrates that the time-consistent optimal strategy is a deterministic function of time if $\rho=0$, and the bigger the $\rho$ is, the more strongly the optimal strategy is disturbed.

5.2 Impact of the exogenous liability on the efficient frontier

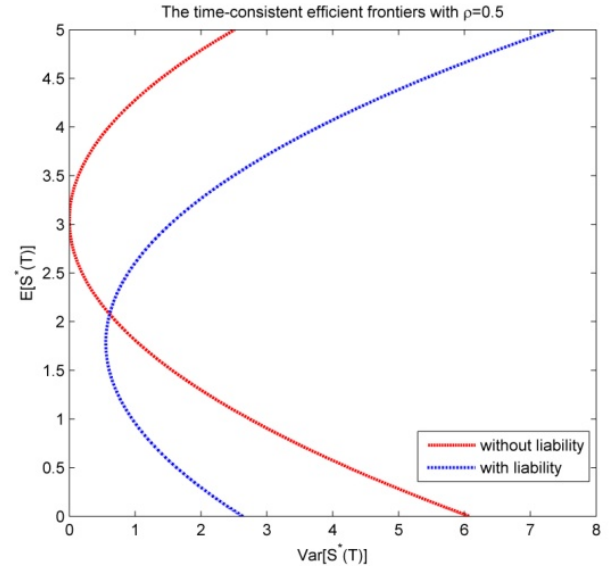

(a)

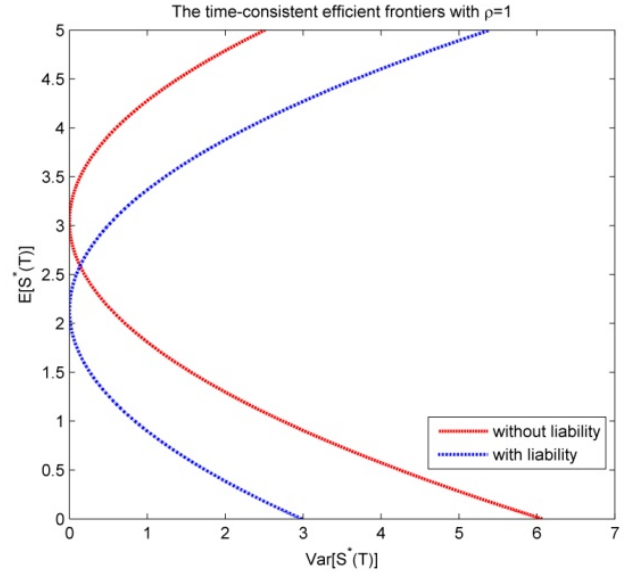

(b)

Figure 2. Impact of the exogenous liability on the time-consistent efficient frontier

In Figure 2, we draw the efficient frontiers under the time-consistent optimal strategies with and without an exogenous liability, respectively. The efficient frontier under the time-consistent optimal strategy with an exogenous liability is drawn according to (36). Note that the correlation coefficient for the efficient frontier with liability in subgraph (a) is chosen as $\rho=0.5$, while the one in subgraph (b) is chosen as $\rho=1$. For the case of no liability, 
letting $L(t)=0, \alpha=0, \beta=0$ and $\rho=0$ for the parameters of (36), the efficient frontier under the time-consistent optimal strategy without liability is derived.

First of all, both subgraphs (a) and (b) show that the efficient frontier under the time-consistent optimal strategy with an exogenous liability is below the one in the case of no liability. This phenomenon agrees with the common sense in that for the same level of expected terminal return, the investor with liability has to invest in more risky asset than the one without liability does, thus the former takes more risk than the latter does.

Second, comparing subgraphs (a) and (b), we find that the efficient frontier with an exogenous liability in subgraph (b) intersects the $\mathrm{E}\left[S^{*}(T)\right]$ axis at one point, while the one in subgraph (a) does not. This is because, unlike the complete correlation of the two Brownian motions which drive the risky asset and the exogenous liability in subgraph (b), the correlation coefficient of these Brownian motions are 0.5 in subgraph (a), thus the risk of the exogenous liability in subgraph (a) can not be completely hedged.

5.3 Comparison of MV efficient frontiers under different optimal strategies

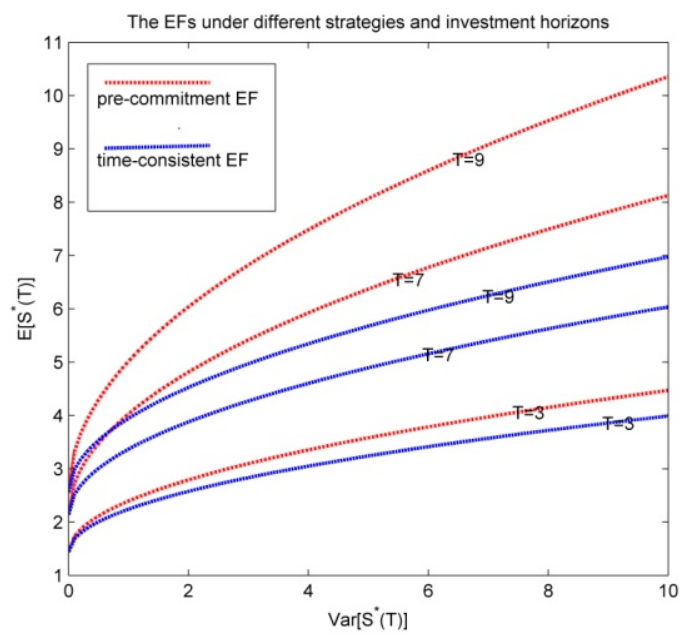

Figure 3. Comparison of the efficient frontier under the time-consistent optimal strategy

with the one under the pre-commitment optimal strategy

In Figure 3, the MV efficient frontier given by (36) is labeled as "time-consistent EF", while the one given by (37) is labeled as "pre-commitment EF". Noted that, according to the model of Chiu and Li (2006), the correlation coefficient is chosen as $\rho=1$.

First of all, no matter what the investment horizon $\mathrm{T}$ is, the efficient frontier under the time-consistent optimal strategy is below the one under the pre-commitment optimal strategy. This is rational because the pre-commitment optimal strategy is the strategy which optimizes the objective function at the initial time, while the time-consistent one can optimize the objective functions at any subsequent time according to the current state. In another word, to derive a time-consistent optimal strategy, we need to take account of additional constraints which are not involved in the MV portfolio selection problem under the pre-commitment mechanism. Obviously, from the perspective of the investor at the initial time, the pre-commitment optimal strategy is more efficient than the time-consistent one.

Second, by comparing the gaps between the time-consistent and the pre-commitment efficient frontier under different investment horizons, we can find that, the longer the investment horizon is, the further the time-consistent efficient frontier is away from the pre-commitment one. The reason is that the longer the investment horizon is, the more decision points the investor has to consider, thus the greater the difference between them is.

\section{Conclusions}

In this paper, we have investigated the time-consistent optimal portfolio strategy for AL management under MV criterion. Specifically, the price of the risky asset and the value of the exogenous liability are driven by different Brownian motions. By using the stochastic dynamic programming approach, the extended HJB equation, the analytical time-consistent optimal strategy, as well as the MV efficient frontier have been derived. Comparing with the model of Basak and Chabakauri (2010) which considers the time-consistent MV portfolio selection problem without liability, and the model of Chiu and Li (2006) which solves the dynamic AL management under MV criterion 
and the pre-commitment mechanism, our work is the first to study the time-consistent optimal portfolio strategy for AL management under MV criterion. Some interesting results are derived: (1) introducing an exogenous liability makes the time-consistent optimal strategy be a stochastic process while the one in the special case of Basak and Chabakauri (2010) is a deterministic one; (2) the efficient frontier of the time-consistent portfolio problem with liability is still a parabola in variance-mean plane; (3) for the efficient frontiers under the time-consistent optimal portfolio strategies, the one for an investor considering an exogenous liability is below the one in which the investor does not take liability into account; and (4) for the continuous-time AL management under MV criterion, the efficient frontier under the time-consistent optimal strategy is below the one under the pre-commitment optimal strategy.

There are still some interesting problems we can study in the future. For instance, (1) This paper assumes that the mean return and the volatility of risky asset are constants. One can consider the case of random mean return or volatility. (2) In our setting, the risk aversion parameter of investor is a constant. It may also be of interest to investigate the case of state dependent risk aversion. (3) It is also worth to consider the case in which the risky asset's price is driven by jump-diffusion process or coefficients of the market depend on the regime of the economy.

\section{Acknowledgements}

We would like to thank the referees for their valuable comments and suggestions. This research is partially supported by NSFC (Nos. 71231008, 71201173), GDUPS (2010), and the High-level Talent Project of Guangdong Province (2011).

\section{References}

Basak, S., \& Chabakauri, G. (2010). Dynamic mean-variance asset allocation. The Review of Financial Studies. 23, 2970-3016. http://dx.doi.org/ 10.1093/rfs/hhq028

Bielecki, T. R., Jin, H., Pliska, S. R., \& Zhou, X. Y. (2005). Continuous-time mean-variance portfolio selection with bankruptcy prohibition. Mathematics Finance. 15, 213-244. http://dx.doi.org/10.1111/j.0960-1627.2005.00218.x

Björk, T., \& Murgoci, A. (2010). A general theory of Markovian time inconsistent stochastic control problems. [Online] Available: http://ssrn.com/abstract=1694759.

Björk, T., Murgoci, A., \& Zhou, X. Y. (2012). Mean variance portfolio optimization with state dependent risk aversion. Mathematical Finance. http://dx.doi.org/10.1111/j.1467-9965.2011.00515.x.

Çakmak, U., \& Özekici, S. (2006). Portfolio optimization in stochastic markets. Mathematical and Methods of Operations Research. 63, 151-168. http://dx.doi.org/ 10.1007/s00186-005-0020-x

Çelikyurt, U., \& Özekici, S. (2007). Multiperiod portfolio optimization models in stochastic markets using the mean-variance approach. European Journal of Operational Research. 179, 186-202. http://dx.doi.org/10.1016/j.ejor.2005.02.079

Chen, P., Yang, H. L., \& Yin, G. (2008). Markowitz's mean-variance asset-liability management with regime switching: A continuous-time model. Insurance: Mathematics and Economics. 43, 456-465. http://dx.doi.org/10.1016/j.insmatheco.2008.09.001

Chiu, M. C., \& Li, D. (2006). Asset and liability management under a continuous-time mean-variance optimization framework. Insurance: Mathematics and 39:330-355. http://dx.doi.org/10.1016/j.insmatheco.2006.03.006

Czichowsky, C. (2013). Time-consistent mean-variance portfolio selection in discrete and continuous time. Finance and Stochastics. 17(2), 227-271. http://dx.doi.org/10.1007/s00780-012-0189-9

Ekeland, I., \& Pirvu, T. A. (2008). Investment and consumption without commitment. Mathematics and Financial Economics. 2, 57-86. http://dx.doi.org/10.1007/s11579-008-0014-6

Karatzas, I., \& Shreve, S. E. (1991). Brownian motion and stochastic calculus. Springer-Verlag: New York.

Koo, H. K. (1998). Consumption and portfolio selection with labor income: A continuous time approach. Mathematical Finance. 8, 49-65. http://dx.doi.org/10.1111/1467-9965.00044

Leippold, M., Trojani, F., \& Vanini, P. A. (2004). A geometric approach to multiperiod mean variance optimization of assets and liabilities. Journal of Economic Dynamics and Control. 28, 1079-1113. http://dx.doi.org/10.1016/S0165-1889(03)00067-8

Li, D., \& Ng, W. L. (2000). Optimal dynamic portfolio selection: Multiperiod mean-variance formulation. Mathematical Finance. 10, 387-406. http://dx.doi.org/10.1111/1467-9965.00100 
Li, Z. F., Zeng, Y., \& Lai, Y. Z. (2012). Optimal time-consistent investment and reinsurance strategies for insurers under Heston's SV model. Insurance: Mathematics and Economics. 51(1), 191-203. http://dx.doi.org/10.1016/j.insmatheco.2011.09.002

Marín-Solano, J., \& Patxot, C. (2012). Heterogeneous discounting in economic problems. Optimal Control Application and Methods. 33(1), 32-50. http://dx.doi.org/10.1002/oca.975

Markowitz, H. (1952). Portfolio selection. Journal of Finance. 7, 77-91. http://dx.doi.org/10.1111/j.1540-6261.1952.tb01525.x

Sharpe, W. F., \& Tint, L. G. (1990). Liabilities-a new approach. Journal of Portfolio Management. 16, 5-10. http://dx.doi.org/10.3905/jpm.1990.409248

Strotz, R. (1956). Myopia and inconsistency in dynamic utility maximization. Review of Economic Studies. 23, 165-180. http://dx.doi.org/10.2307/2295722

Wang, J., \& Forsyth, P. A. (2011). Continuous time mean variance asset allocation: A time-consistent strategy. European Journal of Operational Research. 209, 184-201. http://dx.doi.org/10.1016/j.ejor.2010.09.038

Weiss, N. (2005). A course in probability. Addison-Wesley: Boston.

Xie, S. X. (2009). Continuous-time mean-variance portfolio with liability and regime switching. Insurance: Mathematics and Economics. 45, 943-953. http://dx.doi.org/10.1016/j.insmatheco.2009.05.005

Yi, L., Li, Z. F., \& Li, D. (2008). Multi-period portfolio selection for asset-liability management with uncertain investment horizon. Journal of Industrial and Management optimization. 4, 535-552. http://dx.doi.org/10.3934/jimo.2008.4.535

Yin, G., \& Zhou, X. Y. (2004). Markowitz's mean-variance portfolio selection with regime switching: From discrete-time to their continuous-time limits. IEEE Transactions on Automatic Control. 49, 349-360. http://dx.doi.org/10.1109/TAC.2004.824479

Yong, J. M., \& Zhou, X. Y. (1999). Stochastic controls: Hamiltonian systems and HJB equations. Springer-Verlag: New York.

Zeng, Y., \& Li, Z. F. (2011a). Asset-liability management under benchmark and mean-variance criteria in a jump diffusion market. Journal of Systems Science and Complexity. 24, 317-327. http://dx.doi.org/10.1007/s11424-011-9105-1

Zeng, Y., \& Li, Z. F. (2011b). Optimal time-consistent investment and reinsurance policies for mean-variance $\begin{array}{lllll}\text { insurers. } \quad \text { Insurance: } & \text { Mathematics and }\end{array}$ http://dx.doi.org/10.1016/j.insmatheco.2011.01.001

Zeng, Y., Li, Z. F. \& Wu, H. L. (2013). Optimal portfolio selection in a Levy market with uncontrolled cash flows and only risky assets. International Journal of Control, 86(3), 426-437. http://dx.doi.org/10.1016/j.insmatheco.2011.01.001

Zhou, X. Y., \& Li, D. (2000). Continuous-time mean-variance portfolio selection: A stochastic LQ framework. Applied Mathematics and Optimizations. 42, 19-33. http://dx.doi.org/10.1007=s002450010003

Zhou, X. Y., \& Yin, G. (2003). Markowitz's mean-variance portfolio selection with regime switching: A continuous-time model. SIAM Journal on Control and Optimization. 42, 1466-1482. http://dx.doi.org/10.1137/S0363012902405583

Zhu, S. S., Li, D., \& Wang, S. Y. (2004). Risk control over bankruptcy in dynamic portfolio selection: A generalized mean-variance formulation. IEEE Transaction on Automatic Control. 49, 447-457. http://dx.doi.org/10.1109/TAC.2004.824474 


\section{Appendix}

\section{Proof of Lemma 3.1}

According to Itô's Lemma and the surplus dynamic process (5), we have

$$
\begin{aligned}
& \mathrm{d}\left(\mathrm{S}^{\theta}(t) \exp \left(r_{0}(T-t)\right)\right. \\
& =\exp \left(r_{0}(T-t)\right)\left\{\left[\left(r_{0}-\alpha\right) L(t)+\left(r-r_{0}\right) \theta(t)\right] \mathrm{d} t+\sigma \theta(t) \mathrm{d} W(t)-\beta L(t) \mathrm{d} \tilde{W}(t)\right\} .
\end{aligned}
$$

Assuming that the investor adopts the optimal strategy $\theta^{*}(s)$ in the interval $[t+\tau, T]$, integrating (38) from $t+\tau$ to $T$ and taking the conditional expectation $E_{t+\tau, S^{\theta^{*}}(t+\tau), L(t+\tau)}[\cdot]$ on the both sides of the integration equation derived, we have

which agrees with (12).

$$
\begin{aligned}
& f\left(t+\tau, S^{\theta^{*}}(t+\tau), L(t+\tau)\right) \\
& =\mathrm{E}_{t+\tau, S^{\theta^{*}}(t+\tau), L(t+\tau)}\left[\int_{t+\tau}^{T} \exp \left(r_{0}(T-s)\right)\left[\left(r_{0}-\alpha\right) L(s)+\left(r-r_{0}\right) \theta^{*}(s)\right] \mathrm{ds}\right],
\end{aligned}
$$

2. Proof of Theorem 3.1

According to the recursive formula (10) of $J\left(t, S^{\theta}(t), L(t)\right)$ and the definition of $f\left(t, S^{\theta}(t), L(t)\right)$, we have

$$
\begin{aligned}
& J\left(t, S^{\theta}(t), L(t)\right) \\
& =\max _{\theta(s): s[t, t+\tau]}\left\{\begin{array}{l}
\mathrm{E}_{t, S^{\theta}(t), L(t)}\left[J\left(t+\tau, S^{\theta}(t+\tau), L(t+\tau)\right)\right] \\
-\frac{\lambda}{2} \operatorname{Var}_{t, S^{\theta}(t), L(t)}\left[f\left(t+\tau, S^{\theta}(t+\tau), L(t+\tau)\right)-f\left(t, S^{\theta}(t), L(t)\right)\right. \\
+S^{\theta}(t+\tau) \exp \left(r_{0}(T-t-\tau)\right)-S^{\theta}(t) \exp \left(r_{0}(T-t)\right)
\end{array}\right\}
\end{aligned}
$$

Moving $J\left(t, S^{\theta}(t), L(t)\right)$ to the right side and letting $\tau$ tend to 0 yield the HJB equation in the differential form (13). Combining the definition of $J\left(t, S^{\theta}(t), L(t)\right)$ with $\mathrm{E}_{T, S(T), L(T)}[S(T)]=S(T)$ and $\operatorname{Var}_{T, S(T), L(T)}[S(T)]=0$, the terminal condition is $J(T, S(T), L(T))=S(T)$.

Substituting $S^{\theta}(T)$, which is derived by integrating (38) from $t$ to $T$, into the time $t$ 's objective function $U\left(t, S^{\theta}(t), L(t)\right)$, yields

$$
\begin{aligned}
& \mathrm{E}_{t, S^{\theta}(t), L(t)}\left[S^{\theta}(T)\right]-\frac{\lambda}{2} \operatorname{Var}_{t, S^{\theta}(t), L(t)}\left[S^{\theta}(T)\right] \\
& =S^{\theta}(t) \exp \left(r_{0}(T-t)\right)+\mathrm{E}_{t, S^{\theta}(t), L(t)}\left[\int_{t}^{T} \exp \left(r_{0}(T-u)\right)\left[\left(r_{0}-\alpha\right) L(s)+\left(r-r_{0}\right) \theta(u)\right] \mathrm{du}\right] \\
& -\frac{\lambda}{2} \operatorname{Var}_{t, S^{\theta}(t), L(t)}\left[\begin{array}{l}
\int_{t}^{T} \exp \left(r_{0}(T-u)\right)\left[\left(r_{0}-\alpha\right) L(s)+\left(r-r_{0}\right) \theta(u)\right] \mathrm{du} \\
+\int_{t}^{T} \exp \left(r_{0}(T-u)\right) \sigma \theta(u) \mathrm{dW}(\mathrm{u})-\int_{t}^{T} \exp \left(r_{0}(T-u)\right) \beta L(u) \mathrm{d} \tilde{\mathrm{W}}(\mathrm{u})
\end{array}\right] .
\end{aligned}
$$

According to the above equation, term $S^{\theta}(t) \exp \left(r_{0}(T-t)\right)$ is separable from $L(s)$ and the control $\theta(s)$ for $s \in[t, T]$. Consequently, the optimal strategy $\theta^{*}(s)$ does not depend on $S^{\theta}(t)$ for $s \geq t$.

Furthermore, $\theta^{*}(t)$ depends only on $L(t)$ and $t$ because of the Markovian nature of the economy. Thus, the separable form (14) of the optimal value function $J\left(t, S^{\theta}(t), L(t)\right)$ is derived. Since $f\left(t, S^{\theta}(t), L(t)\right)$ is expressed by (12), it also depends only on $L(t)$ and $t$.

3. Proof of Theorem 3.2

According to the separability property of $J\left(t, S^{\theta}(t), L(t)\right)$ in (14), we have

$$
\mathrm{d} J\left(t, S^{\theta}(t), L(t)\right)=\mathrm{d}\left(S^{\theta}(t) e^{r_{0}(T-t)}\right)+\mathrm{d} \tilde{J}(t, L(t)) .
$$

Applying Itô Lemma to $\tilde{J}(t, L(t))$ and $f(t, L(t))$, we have 


$$
\begin{aligned}
& \mathrm{d} \tilde{J}(t, L(t))=\mathrm{D} \tilde{J}(t, L(t)) \mathrm{d} t+\frac{\partial \tilde{J}(t, L(t))}{\partial L(t)} \beta L(t) \mathrm{d} \tilde{W}(t), \\
& \mathrm{d} f(t, L(t))=\mathrm{D} f(t, L(t)) \mathrm{d} t+\frac{\partial f(t, L(t))}{\partial L(t)} \beta L(t) \mathrm{d} \tilde{W}(t),
\end{aligned}
$$

where $\mathrm{D}$ is an operator which transforms an arbitrary twice continuously differentiable function $F(t, L(t))$ into

$$
\mathrm{D} F(t, L(t))=\frac{\partial F(t, L(t))}{\partial t}+\frac{\partial F(t, L(t))}{\partial L(t)} \alpha L(t)+\frac{1}{2} \frac{\partial^{2} F(t, L(t))}{\partial L(t)^{2}} \beta^{2} L(t)^{2} .
$$

Substituting (41), (42) and (38) into HJB equation (13) yields

$$
\begin{aligned}
& 0=\max _{\theta(t)}\left\{\begin{array}{l}
\mathrm{D} \tilde{J}(t, L(t)) \mathrm{d} t+e^{r_{0}(T-t)}\left[\left(r_{0}-\alpha\right) L(t)+\left(r-r_{0}\right) \theta(t)\right] \mathrm{d} t \\
-\frac{\lambda}{2} \operatorname{Var}_{t, s, l}\left[\frac{\partial f(t, L(t))}{\partial L(t)} \beta L(t) \mathrm{d} \tilde{W}(t)+e^{r_{0}(T-t)} \sigma \theta(t) \mathrm{d} W(t)\right]
\end{array}\right\} \\
& =\max _{\theta(t)}\left\{\mathrm{D} \tilde{J}(t, L(t)) \mathrm{d} t+e^{r_{0}(T-t)}\left[L(t)\left(r_{0}-\alpha\right)+\left(r-r_{0}\right) \theta(t)\right] \mathrm{d} t\right. \\
& -\frac{\lambda}{2}\left\{\left(\frac{\partial f(t, L(t))}{\partial L(t)}\right)^{2} \beta^{2} L(t)^{2}+e^{2 r_{0}(T-t)}\left[\sigma^{2} \theta(t)^{2}+\beta^{2} L(t)^{2}-2 \rho \beta \sigma L(t) \theta(t)\right]\right. \\
& \left.\left.+2 \frac{\partial f(t, L(t))}{\partial L(t)} e^{r_{0}(T-t)} \beta L(t)[\rho \sigma \theta(t)-\beta L(t)]\right\} \mathrm{d} t\right\}
\end{aligned}
$$

The meaning of the above equation is to maximize a quadratic concave function of $\theta(t)$. As a result, the first-order condition of the above optimization problem yields the unique optimal strategy $\theta^{*}(t)$ which is given by (15).

\section{Proof of Theorem 3.1}

Noting that $f(t, L(t))$ is expressed as the conditional expectation form in equation (16), according to Feynman-Kac Theorem (see Karatzas and Shreve (1991) or Yong and Zhou (1999)), $f(t, L(t))$ is the unique solution of following partial differential equation (PDE):

$$
\begin{aligned}
& \frac{\partial f(t, L(t))}{\partial t}+\frac{\partial f(t, L(t))}{\partial L(t)} L(t)\left(\alpha-\frac{r-r_{0}}{\sigma} \beta \rho\right)+\frac{1}{2} \frac{\partial^{2} f(t, L(t))}{\partial L(t)^{2}} \beta^{2} L(t)^{2} \\
& +\left(r_{0}-\alpha+\frac{r-r_{0}}{\sigma} \beta \rho\right) L(t) e^{r_{0}(T-t)}+\frac{1}{\lambda}\left(\frac{r-r_{0}}{\sigma}\right)^{2}=0, f(T, L(T))=0 .
\end{aligned}
$$

Using Feynman-Kac Theorem again, there exists a probability measure $P^{*}$ under which the function $f(t, L(t))$ can be expressed as (17), and under this probability measure, the process $L(t)$ satisfies the following SDE:

$$
\mathrm{d} L(t)=L(t)\left[\left(\alpha-\frac{r-r_{0}}{\sigma} \rho \beta\right) \mathrm{d} t+\beta \mathrm{d} \tilde{W}^{*}(t)\right] .
$$

Comparing the representation formulation (45) with (4), we observe that probability measure $P^{*}$ transforms Brownian motion $\tilde{W}(t)$ into $\tilde{W}^{*}(t)$ in the following form:

$$
\mathrm{d} \tilde{W}^{*}(t)=\tilde{W}(t)+\rho \frac{r-r_{0}}{\sigma} \mathrm{d} t .
$$

We use Girsanov's Theorem to find the Randon-Nikodym derivative $\mathrm{d} P^{*} / \mathrm{d} P$. Recall that the Brownian motion $\tilde{W}(t)$ can be decomposed as a sum of two uncorrelated Brownian motions in the form of the following equation:

$$
\mathrm{d} \tilde{W}(t)=\rho \mathrm{d} W(t)+\sqrt{1-\rho^{2}} \mathrm{~d} W^{0}(t),
$$

where $W^{0}(t)=\frac{\tilde{W}(t)-\rho W(t)}{\sqrt{1-\rho^{2}}}$. Let

It can be observed that

$$
\mathrm{d} W^{*}(t)=\mathrm{d} W(t)+\frac{r-r_{0}}{\sigma} \mathrm{d} t, \mathrm{~d} W^{0^{*}}(t)=\mathrm{d} W^{0}(t)
$$

$$
\mathrm{d} \tilde{W}^{*}(t)=\rho\left(\mathrm{d} W(t)+\frac{r-r_{0}}{\sigma} \mathrm{d} t\right)+\sqrt{1-\rho^{2}} \mathrm{~d} W^{0}(t)=\rho \mathrm{d} W^{*}(t)+\sqrt{1-\rho^{2}} \mathrm{~d} W^{0^{*}}(t) .
$$


Let $Y_{t}=\left(W(t), W^{0}(t)\right)^{\prime}$ and $\Phi(t)=\left(\frac{r-r_{0}}{\sigma}, 0\right)$. According to Girsanov's Theorem, the Randon-Nikodym derivative is

$$
\frac{\mathrm{d} P^{*}}{\mathrm{~d} P}=\exp \left(-\frac{1}{2} \int_{0}^{T}\|\Phi(s)\|^{2} \mathrm{~d} s-\int_{0}^{T} \Phi(s) \mathrm{d} Y_{s}\right)
$$

which coincides with (18).

The remaining work is to derive the analytical optimal strategy $\theta^{*}(t)$. Substituting (17) into (15), we need only to compute $\frac{\partial f(t, L(t))}{\partial L(t)}$. According to (17),

$$
f(t, L(t))=\frac{1}{\lambda}\left(\frac{r-r_{0}}{\sigma}\right)^{2}(T-t)+\int_{t}^{T} \exp \left(r_{0}(T-u)\right) \mathrm{E}_{t, s, l}^{*}[L(u)]\left(r_{0}-\alpha+\frac{r-r_{0}}{\sigma} \beta \rho\right) \mathrm{d} u .
$$

Let $g(t, u)=\mathrm{E}_{t, s, l}^{*}[L(u)]$, for $u \geq t$. Since

$$
\mathrm{d} L(t)=L(t)\left(\alpha-\frac{r-r_{0}}{\sigma} \beta \rho\right) \mathrm{d} t+\beta L(t) \mathrm{d} \tilde{W}^{*}(t),
$$

integrating the above equation from $t$ to $u$ and taking the conditional expectation $\mathrm{E}_{t, s, l}^{*}[\cdot]$ on the both sides of the integration equation derived, we obtain the representation of $g(t, u)$ as

$$
g(t, u)=L(t)+\int_{t}^{u} g(t, s)\left(\alpha-\frac{r-r_{0}}{\sigma} \beta \rho\right) \mathrm{d} s .
$$

Differentiating (47) with respect to $u$ yields

$$
\frac{\partial g(t, u)}{\partial u}=g(t, u)\left(\alpha-\frac{r-r_{0}}{\sigma} \beta \rho\right)
$$

with initial condition $g(t, t)=L(t)$. After calculation, the unique solution of ODE (48) is

$$
g(t, u)=L(t) \exp \left(\left(\alpha-\frac{r-r_{0}}{\sigma} \beta \rho\right)(u-t)\right) .
$$

Substituting (49) into (46), we have

$$
f(t, L(t))=\frac{1}{\lambda}\left(\frac{r-r_{0}}{\sigma}\right)^{2}(T-t)+L(t)\left[\exp \left(r_{0}(T-t)\right)-\exp \left(\left(\alpha-\frac{r-r_{0}}{\sigma} \beta \rho\right)(T-t)\right)\right] .
$$

Finally, substituting (50) into (15) yields the $\theta^{*}(t)$ represented in (19). 